\title{
Fernsehunterhaltung bis zum Jahr 2000. Im Spannungsfeld zwischen Kulturverfall und Alltagskultur
}

\author{
von Dieter Stolte
}

Im Spannungsfeld von „Alltagskultur“ und „Kulturverfall“ ist der Stellenwert der Fernsehunterhaltung ein Thema, das von einer eindeutigen Definition dessen ausgeht, was Kultur ist oder sein sollte. „Die“ Definition gibt es jedoch ebensowenig wie es einen allgemeingültigen Maßstab für, guten' Geschmack oder 'gute' Unterhaltung geben kann. Deshalb möchte ich unter Umgehung von Geschmacksfragen versuchen, im ersten Teil dieses Beitrags darzustellen, was in meinen Augen Fernsehunterhaltung zu einem integralen Bestandteil des Fernsehens macht, und im zweiten Teil darauf eingehen, wie Fernsehunterhaltung heute aussieht und wie sie sich in Zukunft entwickeln muß, wenn sie nicht zum vielbeschworenen Kulturverfall führen soll.

\section{Die Funktion der Unterhaltung als Spiel}

Um zu verstehen, was Unterhaltung eigentlich mit Fernsehen zu tun hat, ist ihre Funktion zu klären. Neben umfassender Information und angemessener Bildung sucht der Zuschauer im Fernsehen in erster Linie Unterhaltung und Entspannung, um sich von den Anstrengungen des Alltags zu erholen. Wenn man sich fragt, was Unterhaltung eigentlich ist, sollte man mit einem Umkehrschluß beginnen: Unterhaltung ist das Gegenteil von Langeweile. Ihr Wert bemißt sich nicht nach inhaltlichen oder formalen Kriterien, sondern am Interesse der Unterhaltenen.

Unterhaltung als Gegenpol der Langeweile bezeichnet ein Genre, das im Land der „Dichter und Denker“ gern der Trivialität verdächtigt wird. Dabei ist sie als Grundbedürfnis für ein menschenwürdiges Leben ebenso notwendig wie der Humor oder das Lachen, denen man aber gerne wegen mangelnder Ernsthaftigkeit ihre Bedeutung abspricht. ${ }^{1}$ Aber auch die Liste der Verteidiger der Unterhaltung - und sei es als Inbegriff des Spieltriebes - ist lang und reicht von Schiller, der den Spieltrieb seiner Zweckfreiheit wegen als das eigentlich Humane bezeichnet, bis hin zu Johan Huizinga, der in seinem Werk „Homo ludens" die These vertritt, daß „Kultur" nicht im Ernst, sondern aus dem Spiel entsteht. ${ }^{2}$ Und schließlich haben Philosophen von Aristoteles bis Helmuth Plessner versucht, den Menschen gerade durch das zu definieren, was ihn vom Tier unter-

Prof. Dr. h.c. Dieter Stolte ist Intendant des Zweiten Deutschen Fernsehens.

1 Vgl. Luigi Pirandello, L' umorismo; deutsch: Der Humor, Essay, aus dem Italienischen von Johannes Thomas, Mindelheim 1986, 60, der Rabelais zitiert: „Pour ce que le rire est le propre de l' homme“ (Denn das Lachen ist dem Menschen eigen). Siehe insbesondere Helmuth Plessner, Lachen und Weinen, München 1950, 13.

2 Johan Huizinga, Homo ludens, Vom Ursprung der Kultur im Spiel (1938). Neuauflage Hamburg 1956, 34: „Spiel ist eine freiwillige Handlung oder Beschäftigung, die .... ihr Ziel in sich selber hat und begleitet wird von einem Gefühl der Spannung ..." 
scheidet: Die Fähigkeit zu lachen - und sich damit gut zu unterhalten. Daß dies sich auch in der Tradition niedergeschlagen hat, läßt sich an den Werken Shakespeares nachvollziehen: Obwohl oder gerade weil sie spielerisch und unterhaltsam mit den Problemen der Welt und des Lebens umgehen, können ernste Themen besonders eindrucksvoll dargestellt werden. Im Spiel wird aus der natürlichen Wirklichkeit ein Spielraum ausgegrenzt, das Spiel wird gerade dadurch attraktiv, daß es Überfluß ist. „Überflüssig“ ist es jedoch nur in dem Sinne, der einer ansonsten von Zwecken beherrschten Gesellschaft zugrundeliegt. Spielerische Unterhaltung jedoch will in erster Linie nichts anderes als unterhalten. Fiktives, Phantastisches und Utopisches ist im Spiel: „Fernseh-Spiel“ oder "Spiel-Film“ sind nicht mehr an der Realität, sondern an ihrem Unterhaltungswert zu messen. Unterhaltung geht jedoch auch im Spiel über das bloß Spielerische hinaus, wenn es ihr gelingt, anregend zu wirken, Spannung wie Entspannung zu ermöglichen. ${ }^{3}$ Gerade dieser Mangel an tierischem Ernst jedoch ist es, der den Wert der Unterhaltung ausmacht: Indem sie nicht vorgibt, etwas zu sein, was sie nicht ist (nämlich „hochkulturell“ oder „nützlich“), gelingt es ihr spielerisch, neue Ebenen zu erschließen. Gerade im unterhaltenden, spielerischenGespräch entstehen neue Ideen, wird Kreativität angeregt und gefördert. Natürlich kann Unterhaltung auch realitätsbezogene und zeitkritische Elemente enthalten - aber in erster Linie muß sie dem zu Unterhaltenden, hier dem Zuschauer, gefallen.

\section{Die drei Funktionen der Fernsehens}

Die klassische Aufteilung der Funktionen des Fernsehens in Information, Bildung und Unterhaltung findet sich als Aufgabenstellung bereits in den „Richtlinien für die Sendungen des ZDF“:

"Das Programm soll umfassend informieren, anregend unterhalten und zur Bildung beitragen. ${ }^{\star 4}$

Diese Verbindung von informativ-bildender und unterhaltender Funktion der Medien ist keineswegs eine Erfindung des Fernsehzeitalters. Für das Buch hat Horaz festgestellt: ,Jede Stimme erhält, wer Süßes und Nützliches mischte, indem er den Leser ergötzte und gleicherweise belehrte. "S Übertragen auf den Zuschauer folgt daraus auch heute noch, daß die Verteilung der einzelnen Programmbestandteile auf die Funktionen Bildung, Information und Unterhaltung nie pauschal erfolgen kann: Jede Sendung, die wirkungsvoll bilden will, muß auch informative und unterhaltende Elemente enthalten - so daß keines der

3 Vgl. Hans Kimmel, Schein und Sein. Ein Plädoyer gegen den ständigen Ernstfall, in: Gerhard Prager (Hg.), Fernseh-Kritik, Unterhaltung und Unterhaltendes im Fernsehen, Mainz 1971, 87-102.

4 Siehe schon die „Richtlinien für die Sendungen des „Zweiten Deutschen Fernsehens“ vom 11. Juli 1963, abgedr. in: Ernst W. Fuhr, ZDF-Staatsvertrag, Mainz 1985, 460.

5 Siehe Horaz, Ars Poetica/Die Dichtkunst, Vers 334. Vgl. auch „omne tulit punctum, qui misuit utile dulci“ (der hat den größten Erfolg, der gefällig und nützlich zugleich). 
Genres getrennt bestehen kann, wenn man ein vom Publikum akzeptiertes Qualitätsfernsehen anstrebt.

Eine lange Tradition des Kulturelitismus hat es sich allerdings zur Aufgabe gemacht, aus dieser Trias das Element der Unterhaltung als minderwertig zu verbannen - und versucht, die öffentlich-rechtlichen Fernsehanbieter als Hüter der Hoch-Kultur alleine auf die Bildungs- und Informationsfunktion festzulegen und als Form der Arbeitsteilung den kommerziellen Programmen das massenattraktive Unterhaltungsprogramm allein zu überlassen. Ich wende mich wie Hermann Bausinger in seinem Beitrag in diesem Heft gegen den „Rufmord an der Unterhaltung" - und halte sie für ein unverzichtbares Element jedes erfolgreichen Fernsehprogramms, das auch nach dem Jahr 2000 noch höhere Zuschauerzahlen haben will als das werbe- und unterhaltungsfreie, an den publizistischen Rand des Interesses gedrängte „PBS“ der U.S.A.. Um dies ${ }^{*}$ zu erläutern und das Übergreifen amerikanischer Verhältnisse von Kulturverfall durch Unterhaltung zu verhindern - ist eine Differenzierung der verschiedenen Kultur- und Unterhaltungsbegriffe notwendig.

\section{Drei Kulturbegriffe im Fernsehen}

Was ist Kultur, und was kann Fernsehen zur Kultur beitragen? Schließen sich Kultur und Unterhaltung gegenseitig aus oder wird die eine von der anderen verdrängt? Die Antwort auf diese Fragen ist zugleich der Schlüssel zur Antwort auf die Frage nach der Rolle der Unterhaltung im Fernsehen. Im wesentlichen sind drei Kulturbegriffe unterscheidbar:

- Als erstes ist auf die Elite- oder Hochkultur zu verweisen, wie sie sich in den sogenannten „schönen Künsten“ findet. Hierunter zählt man ebenso anspruchsvolle Literatur oder Philosophie, „ernste“ Musik wie Oper oder auch Malerei und Bildhauerei. Als Werk weniger Auserwählter für eine gebildete Elite hat dieser Kulturbegriff im Zeitalter des Absolutismus seine Blüte erlangt.

- Zweitens ist auf den Kulturbegriff des Humanismus zu verweisen, der als Mischung von Bildung und Urbanität den ganzen Bereich höheren menschlichen Schaffens umschließt: Neben die eben genannten „bildenden Künste“ treten hier alle Hervorbringungen des Menschen, also auch der politische und der wissenschaftlich-technische Bereich. Der Fortschritt der Gesellschaft wird in dieser pragmatischen Auffassung zum Teil der kulturellen Entwicklung.

- Schließlich ist von diesen beiden Kulturbegriffen das Konzept der Kultur als Massenkultur zu unterscheiden, die den Kulturbegriff so erweitert, daß alles Populäre hierunter subsumiert werden kann: Von der Popmusik über Mode bis zum Kitsch reicht die Palette, die sich je nach Zeitgeschmack ständig erweitert. Von den Vertretern der Elitekultur wird diese Form abwertend gerade durch ihren Unterhaltungswert als niedere Kultur eingestuft und abgelehnt. 
Kulturbegriffe sind historisch entstanden und können nicht starr beibehalten werden. Auch und gerade die deutsche Kultur ist nicht starr, sondern dynamisch, wie das Zusammenwachsen der beiden Teile Deutschlands anschaulich belegt. Es liegt im Wesen einer lebendigen Kultur, Neues hervorzubringen, Altes zu verwerfen, sich zu erneuern. Nur als sich Erneuernde ist sie lebensfähig - und das Fernsehen ist als Teil, Medium und Faktor der Kultur charakteristisch für die Gegenwart und ihren Wandel.

Eigentümlich ist allen drei Kulturbegriffen, daß sie nebeneinander und sich ergänzend, allerdings in verschiedenen Gesellschaftsschichten, fortbestehen. Es handelt sich ${ }^{*}$ jedoch nicht um ein friedliches Nebeneinander, da sich Begriffe wie Hoch- und Massenkultur auszuschließen scheinen - und Vertreter aller Richtungen der jeweils anderen Sichtweise die Qualifikation absprechen. Während einerseits gerade durch die modernen Massenmedien die Internationalisierung der Kultur um sich greift, versuchen andererseits kulturelle Minderheiten, dem damit gleichgesetzten Niveauverlust Einhalt zu gebieten, indem sie in Opern, Literatur oder Konzerten die Hochkultur, die auch eine Kultur des Buches war, gegen Popularisierung verteidigen.

Das Fernsehen befindet sich nun in dem Dilemma, allen Kulturbegriffen verpflichtet zu sein - und wirklich finden bis heute, wenn auch in verschiedenem Maße, alle Kulturformen in den Programmen ihren Platz. Eine Entscheidung für den einen und gegen den anderen Kulturbegriff ist unmöglich - und wird doch gefordert, wenn mit Hinweis auf die Niveaulosigkeit oder Gefährlichkeit von Unterhaltung verlangt wird, Unterhaltung als Massenkultur ganz vom Bildschirm zu verbannen - oder doch wenigstens den Kommerzsendern zu überlassen, um sich wieder auf die hehre, unterhaltungsfreie Hochkultur konzentrieren zu können. Dieser Streit wird auf dem Rücken der Unterhaltung ausgetragen.

\section{Die Unterhaltungsfunktion des Fernsehens als Massenmedium}

Nachdem wir bereits die Funktion der Unterhaltung allgemein zu klären versucht haben, ist jetzt auf die Unterhaltungsfunktion im Massenmedium einzugehen. Vom elitären Blickwinkel gehört es zu den Allgemeinplätzen der neueren deutschen Kulturkritik, den angeblichen Kulturverfall durch ihre Popularität mit der Fernsehunterhaltung in Verbindung zu setzen. Gestützt wird sie dabei von der durch Neil Postman vertretenen These, daß wir uns nach dem beklagenswerten Niedergang des Buchdruckzeitalters heute durch das Fernsehen zu Tode amüsieren. ${ }^{6}$

Eine gängige These lautet, gerade die Fernsehunterhaltung werde immer niveauloser und müsse durch bessere, das heißt kulturell höherstehende Pro-

6 Vgl. Neil Postman, Wir amüsieren uns zu Tode, Frankfurt/M. 1985, bes. 12-17. Siehe dagegen meine Ausführungen zum Nebeneinander von Unterhaltung und Kultur in: Fernsehen am Wendepunkt - Meinungsforum oder Supermarkt? (in Zusammenarbeit mit E. Kettering), München 1992, bes. 69 ff. u. 87. 
gramme ersetzt werden. Als mögliche Antworten auf diesen Vorwurf des $\mathrm{Ni}$ veauverlusts in den breitenwirksamen Unterhaltungssendungen der Hauptprogramme könnte die Entwicklung von reinen Kulturprogrammen wie „ARTE“ gesehen werden - die jedoch mit Marktanteilen um die Ein-Prozent-Marke nur eine kleine Minderheit der Bevölkerung erreichen. Sind aber anspruchsvolle reine Kulturprogramme allein die richtige Lösung des Konflikts zwischen anspruchsvoller Hoch-Kultur für wenige einerseits und anspruchslosem Entertainment für viele andererseits?

Ein Denkfehler führt zu dieser Scheinalternative. In einem kulturellen Ghetto versucht man, den elitären Kunstbegriff gegen den Zeitgeist zu schützen und übersieht, daß sich der Kulturbegriff, nicht zuletzt durch das Fernsehen, heute erweitert hat. Zur Kultur gehören eben nicht mehr nur Dichtung, Oper und klassische Musik, sondern ebenso die Kultur des täglichen Lebens, die von der Mundart bis zum Kabarett und vom Sport bis zur Fernsehshow reicht. Nicht Trennung der Genres, sondern die Organisation eines möglichst harmonischen Zusammenwirkens aller Gattungen in einem Programm ist Ziel einer zeitgemäßen Programmpolitik jedes Vollprogramms im Fernsehen. Daneben kann es wenn es Interessenten gibt, die bereit sind, dafür auch entsprechend zu zahlen auch Special-Interest-Programme für Elitekultur und -unterhaltung geben. Es sollte nie vergessen werden, daß Fernsehen im Gegensatz zu den Printmedien, die auch kleinere Minderheiten versorgen können, von Anfang an ein Massenmedium, also ein Medium für breite Zuschauerschichten, war. Hierfür gibt es drei Gründe:

- Der erste ist rein technischer Natur: Die Verbreitung des Fernsehens, sei es terrestrisch, via Kabel oder Satellit, übersteigt die Möglichkeiten aller vorangegangenen Medien.

- Der zweite Grund ist rechtlicher Natur: In einer Demokratie sind Programme grundsätzlich allen Zuschauern zugänglich zu machen, die dies wünschen. Spartenprogramme sind hiervon keine Ausnahme, selbst wenn es sich um „Pay-per-view“-Programme handelt. Auch diese müssen um möglichst große technische Reichweiten bemüht sein.

- Der letzte - und immer wichtiger werdende - Grund ist ökonomischer Natur: Dies gilt ebenso für den Bereich der sich ständig verteuernden technischen Ausrüstung, die zur Produktion und Verbreitung der Programme benötigt wird, wie für die Inhalte. Eine ständig steigende Zahl von Sendern macht sich ein immer knapper werdendes Angebot an Filmen, Darstellern, Ereignissen oder Inhalten streitig. Die sich aus der Verknappung des Angebots und der Steigerung der Nachfrage ergebende Preissteigerung zwingt die Sender dazu, sich eine möglichst breite Zuschauerschaft zu sichern.

Wenn man sich diese Definition des Fernsehens als Massenmedium vergegenwärtigt, sieht man, daß ein elitäres Programm als Hauptprogramm einen Widerspruch in sich darstellt. Nur wenn es gelingt, auch kulturellen Programmen (die selbst weniger der Preisspirale unterworfen sind als Unterhaltungssendungen) ein relativ breites Publikum zu verschaffen, können sich diese beim Gebührenzahler legitimieren - oder beim für sie zahlenden Kunden durchset- 
zen. Auch Minderheitenprogramme müssen innerhalb der angesprochenen Zielgruppe eine Mehrheit der potentiellen Zuschauer erreichen, wollen sie auf Dauer im Massenmedium Fernsehen bestehen. ${ }^{7}$ Für reine Bildungsprogramme ohne jeden Unterhaltungswert sehe ich in der durch die Vielzahl der Kanäle bestimmten Medienlandschaft heute und in Zukunft keine Chance mehr, mit nachweisbarer Akzeptanz wahrgenommen zu werden. Die Notwendigkeit, sich auch in einer sich immer stärker nach verschiedenen Interessen differenzierenden Zuschauerschaft an ein relativ breites Publikum wenden zu müssen, um finanzierbar zu bleiben, betont heute den Wert der für alle Zuschauer attraktiven unterhaltenden Elemente in allen Programmformen mehr denn je. Elitäre Ansprüche passen nicht zum Fernsehen als Massenmedium.

Das ,Geheimnis' des Massenmediums, sich immer ein breites Publikum suchen zu müssen, auch wenn man von vornherein weiß, daß es ein homogenes Massenpublikum gar nicht gibt, läßt sich für eine erfolgreiche Programmpolitik nur dadurch lösen, daß die verschiedenen Teile der Gesellschaft gleichermaßen angesprochen werden müssen - und Interesse auch für diejenigen Programmteile zu wecken ist, die nicht ohne weiteres jeden Fernsehzuschauer interessieren. Gerade hier liegt die große Chance der Unterhaltung, wenn es ihr gelingt, auch innerhalb publikumsattraktiver Unterhaltungsprogramme bildende und informative Elemente einzubringen.

Um jedoch nicht von einem Extrem ins andere zu fallen, ist es nötig, nach der Ablehnung eines Elite-Fernsehens auch eine einseitige Sicht des Fernsehens als reine Unterhaltungsmaschine der Populärkultur zu vermeiden, ${ }^{8}$ die alleine den Geschmack der Masse der Zuschauer zum Kulturkriterium erhebt.

\section{Alltagskultur im Fernsehen}

Um keine Abwertung vorzunehmen und anzudeuten, daß eine Entscheidung für die eine Kultursicht und gegen die andere heute mehr denn je unmöglich ist, habe ich vor Jahren den Begriff der „Alltagskultur“ geprägt, der bereits im Begriff die Berührungspunkte zwischen Elitär- und Popularkultur verdeutlicht. ${ }^{9}$ Wer wüßte heute schon genau, wo die Trennungslinie zwischen sogenannter anspruchsvoller „E-Musik“ und populärer „U-Musik“ zu ziehen wäre? Darf man sich bei einem Bach-Konzert nicht ebensogut unterhalten fühlen wie

7 In Relation zu den begrenzten Möglichkeiten traditioneller Kulturinstitutionen wie dem Theater stellt auch eine Einschaltquote von einem Prozent für ca. $500.000 \mathrm{Zu}$ schauer eine Masse dar. Die Masse bemißt sich am Potential interessierter Zuschauer, so daß sie hier mit den Einschaltquoten populärer Großereignisse wie Fußballspielen von ca $30-40 \%$ vergleichbar sind.

8 Wie Theodor W. Adorno dies mit seiner Kritik an der in seinen Augen nur konsumorientierten "Massenkultur" als Unkultur versuchte. Vgl. Television and the Patterns of Mass Culture, in: B. Rosenberg, Mass Culture Revisited (hg. v. D. M. White), New York 1971, 123, sowie Ursula Dehm, Fernsehunterhaltung. Zeitvertreib, Flucht oder Zwang?, Eine sozialpsychologische Studie zum Fernseherleben (Kommunikationswissenschaftliche Bibliothek, Band 10), Mainz 1984, $57 \mathrm{ff}$.

9 Vgl. meinen Aufsatz „Kultur für alle“, in: Die Zeit, Nr. 31 vom 27.06.84, 
bei einem Rock-Konzert, ohne dabei den Ernst der Kultur zu vernachlässigen? Auch gesellschaftlich haben sich Grenzen verschoben: derselbe Zuschauer ist Anhänger populärer Sportsendungen wie elitärer Kulturprogramme, nur eben alles zu seiner Zeit.

Umberto Eco hat hierzu eine Definition der Alltagskultur geprägt, die meines Erachtens auch für ein gutes Fernsehprogramm (sei es kommerziell oder öffentlich-rechtlich) gilt:

"Das Problem einer ausbalancierten kulturellen Kommunikation besteht nicht darin, diese Botschaften abzuschaffen, sondern darin, sie zu dosieren - und zu vermeiden, daß sie als (elitäre) Kunst verkauft und rezipiert werden." 10

Gelungene Fernsehkultur spiegelt in sich auch die Programmvielfalt wider, indem sie sich zwar nach den Wünschen und den Bildungsvoraussetzungen jener $80 \%$ richtet, welche die Masse seiner Zuschauer ausmachen, aber trotzdem seine kulturellen und informativen Pflichten nicht vernachlässigt. Das heißt, daß einerseits neben unterhaltenden immer auch bildende und informative Elemente in allen Sendungen enthalten sein müssen, und andererseits, daß auch den wechselnden Minderheiten der Liebhaber von Theater, anspruchsvollen Büchern oder Konzerten spezifische Programmangebote zu machen sind. Programmvielfalt in Art und Anspruch ist eine Möglichkeit, auch bei einer sich in verschiedene Interessengruppen aufsplitternden Öffentlichkeit noch ein breites Publikum anzusprechen. Eine Verbindung verschiedener Teile der Zuschauerschaft, die keine Vermischung ist, kann nicht in allen Programmen gelingen und bildende Programmteile, die dem elitären Kulturbegriff entsprechen, sind zwar wichtig, aber eben per definitionem nicht in der Lage, breite Zuschauerschichten anzulocken. Trotzdem kann es einer am Begriff der Alltagskultur ausgerichteten Programmpolitik gelingen, auch hierfür breiteres Interesse zu wekken, indem massenattraktive Unterhaltungsprogramme den Blick der Zuschauer auch auf andere Kulturkreise lenken. Alltagskultur im Fernsehen verbindet nicht nur die 3 Kulturbegriffe, sondern auch die 3 Funktionen des Fernsehens. Der Forderung nach Kultur mit hohem Anspruch oder pragmatischer Lebenshilfe entsprechen die Bereiche der Bildung und der Information, während die Pflege der Massenkultur mit dem Bereich der Unterhaltung korrespondiert. Die Funktionen ergänzen sich dabei im Idealfall wechselseitig.

Von interessierter Seite, sei es in der Politik oder den privaten Medien, wird allerdings vorgeschlagen, in Zukunft die massenattraktive Unterhaltung den gewinnorientierten kommerziellen Sendern allein zu überlassen, und das dann ausschließlich gebührenfinanzierte öffentlich-rechtliche Fernsehen auf Bildung und Information zu beschränken. ,Übersehen' wird bei diesem Vor-

10 Vgl. Umberto Eco, Die Struktur des schlechten Geschmacks, in: ders., Apokalyptiker und Integrierte. Zur kritischen Kritik der Massenkultur, Frankfurt/M. 1984, 73 (Hervorhebung von mir). 
schlag, daß der Begriff der Alltagskultur von einer wechselseitigen Durchdringung der Programmelemente ausgeht.

Unterhaltung ist gerade dann, wenn sie spielerisch vorzugehen weiß, besonders gelungen. Nur dann kann es gelingen, ernste Elemente unterhaltsam einzubauen und den erhobenen Zeigefinger eines elitären Bildungsfernsehens zu vermeiden. Werke wie Goethes „Werther" wurden gerade dadurch zur Weltliteratur, daß sie ernste und unterhaltende Elemente in packender Form miteinander verbanden. Ähnliches kann auch heute für die Fernsehunterhaltung gelten: Eine erfolgreiche „Knoff-hoff-Show“ oder Filme wie „Der Große Bellheim“ zeigen, daß qualitätsorientierte Unterhaltung der verschiedensten Genres im Massenmedium Fernsehen erfolgreich sein kann. Unterhaltungsfernsehen kann ebenso wie Unterhaltungsliteratur Massenware für den Augenblick sein - aber sie muß es nicht. Alltagskultur ist auch Gebrauchskultur; Gebrauchskultur in dem Sinne, daß das tägliche Leben nicht nur aus Ernst, Bildung und Information bestehen kann, sondern notwendig auch Unterhaltung und Entspannung beinhalten muß. Zur Gefahr wird Unterhaltung erst dann, wenn sie sich in alle Bereiche des Massenmediums Fernsehen einnistet und den Zuschauer zum passiven, isolierten Unterhaltungsvielseher degradiert. Um dies zu verhindern, können auch gerade qualitativ hochstehende und trotzdem breitenwirksame Unterhaltungsprogramme einen wichtigen Beitrag leisten. Kriterium für ihre Bewertung ist einerseits das Ausbleiben von Langeweile - und der im Rückblick auf eine hoffentlich unterhaltsam verbrachte Zeit erfahrene Gewinn: Einen amüsanten und entspannenden Fernsehabend verbracht zu haben.

Unter Einbeziehung gerade der entspannenden Funktion der Unterhaltung hat das Bundesverfassungsgericht den Begriff der Grundversorgung geprägt, die durch die öffentlich-rechtlichen Anbieter zu leisten ist.

\section{Unterhaltung als Teil des Grundversorgungsauftrags und ,Grundver- sorgung' mit Gefühlen}

Indem das Bundesverfassungsgericht seit 1986 in seinem 4. Rundfunkurteil den öffentlich-rechtlichen Rundfunkanstalten den Grundversorgungsauftrag zuwies, bestätigte es die Bestandsgarantie für den öffentlich-rechtlichen Rundfunk im dualen System.

Diese Position wird von kommerzieller Seite mit dem Hinweis darauf in Frage gestellt, daß es mittlerweile private Vollprogramme gäbe, die ihrerseits in der Lage wären, Teile der Grundversorgung zu übernehmen. Damit verbunden wird die bereits genannte Forderung, in Zukunft öffentlich-rechtliche Programme auf den kulturellen und bildenden Teil zu beschränken, während das massen- und werbeattraktive Unterhaltungsprogramm für kommerzielle Anbieter zu reservieren sei. Hier wird versucht, die Unterhaltung aus dem Grundversorgungsauftrag herauszulösen bzw. eine Trennung der Programme nach Zielgruppen vorzunehmen. 
Dem steht allein schon die Definition entgegen, die das Bundesverfassungsgericht in seinem Werbeverbots-Urteil zum Regionalprogramm Hessen 3 am 6. Oktober 1992 geprägt hat: „Grundversorgung bedeutet ... weder eine Mindestversorgung noch beschränkt sie sich auf den informierenden und bildenden Teil des Programms." Sie ist vielmehr eine Versorgung mit Programmen, die dem klassischen Rundfunkauftrag entsprechen, wie ihn das Bundesverfassungsgericht mehrfach definiert hat, und die technisch für alle empfangbar sind. Entscheidet sich der Gesetzgeber für ein duales System, muß er die Voraussetzungen für die Erbringung dieser Leistung in jeder Hinsicht, auch finanziell, sicherstellen.

Ohne an dieser Stelle tiefer in die Grundversorgungsdebatte einzusteigen kann festgestellt werden, daß die Grundversorgung, die ausdrücklich den Unterhaltungsauftrag mit einschließt, als Pflicht nur den öffentlich-rechtlichen Fernsehveranstaltern auferlegt wurde. Andererseits läßt sich daraus ableiten, daß auch bei Erfüllung von Teilen des Grundversorgungsauftrages durch Privatveranstalter diese - jederzeit änderbare - Programmpolitik „Gegenstand privatrechtlicher Beliebigkeit ist, nicht treuhänderischer Pflichtigkeit" ${ }^{\text {" }}{ }^{11}$ Würde auch und gerade im Unterhaltungssektor - die zum Niveau verpflichtete Konkurrenz der öffentlich-rechtlichen Anbieter fehlen, wäre ein weiteres Absinken des Niveaus kommerzieller Programme zu erwarten.

Neben die juristische Pflicht zur Grundversorgung tritt ein Bedürfnis, das man als ,Grundversorgung mit Gefühlen' bezeichnen könnte. Wenn Serien "gut“ unterhalten, d.h. den Zuschauer ansprechen und sogar zum Träumen anregen, nehmen sie die Wünsche der Zuschauer ernst. Zwar mögen Serien vom Glottertal bis zum Traumschiff nicht dem Geschmack einer am elitären Kulturbegriff orientierten Fernsehkritik entsprechen - aber für gefährlich wird sie ernsthaft niemand halten können. Wichtig ist, daß auch hier Probleme des Alltags nicht völlig ausgeklammert und Ansätze zu ihrer Lösung aufgezeigt werden. Nicht daß der Serienheld zum Vorbild wird, ist das Problem, sondern zu welchen Vorbildern man sich bekennt; und hier halte ich nach wie vor Pfarrer und Chefärzte für annehmbarer als kriegerische Helden à la Rambo, die nur mit Mitteln der Gewalt Konflikte lösen können. Gerade eine beliebte Vorabendserie wie „Unser Lehrer Dr. Specht" kann durch einen realgesellschaftlichen Rahmen hohe Einschaltquoten und gesellschaftlichen Auftrag des Fernsehen aufs Beste verbinden. Gerade unterhaltend ist es so möglich, Tabus und Probleme wie Rechtsradikalismus oder die Differenz Ost/West anzusprechen und wichtige Inhalte mitzuteilen.

Die ,Grundversorgung mit Gefühlen' kann auch zum aktiven Engagement der Zuschauer führen, wenn sich Unterhaltung nicht nur auf den ablenkenden Zeitvertreib beschränkt. Mitgehen und Mitfühlen sind durch die Unterhaltung zu aktivieren und das soziale Engagement der Zuschauer zu wecken. Hinweisen möchte ich in diesem Zusammenhang auf die mit Unterhaltungssen-

11 Vgl. Herbert Bethge, in: Reinhold Kreile (Hg.), Medientage München 1992, Dokumentation, Baden-Baden 1992. 
dungen wie "Der große Preis“ erfolgreich genutzten Chancen, für die Aktion Sorgenkind über 2 Milliarden DM für einen guten Zweck zu sammeln - und gleichzeitig in Form eines Quiz Bildungselemente zu integrieren. Hier liegt die Einheit von qualtiätsbewußter Unterhaltung, dadurch hoher Einschaltquote und gutem Zweck vor. Die „Aktion Sorgenkind“ steht zugleich beispielhaft für das Selbstverständnis und die Leistungskraft des öffentlich-rechtlichen Systems.

Daneben hat aber auch die, reine' Unterhaltung einen berechtigten Anspruch auf Sendeplätze: Auch wenn Unterhaltungssendungen wie z.B. „Traumhochzeit" keinen sozialen Zweck verfolgen, befriedigen sie das legitime Bedürfnis der Zuschauer nach Unterhaltung. Die Genres sind vielfältig und umfassen ebenso das anspruchsvolle kleine oder große Fernsehspiel, die unterhaltsame Diskussionsrunde des „Literarischen Quartetts“, die spannende Unterhaltung bei einem Krimi, den entspannenden Genuß einer Fernseh-Show oder ein pakkendes Fußballspiel. Schon in der Sparte „Musik" reicht der Bogen von der live gesendeten Opernaufführung „La Traviata“ über die Ausstrahlung von Pop-Konzerten oder Jazz-Festivals bis hin zur Folklore oder der „Hitparade der Volksmusik". Diese willkürlich aufgegriffenen Beispiele, die alle Unterhaltung für verschiedene Zuschauerschichten bieten, legitimieren sich durch ihre Erfolge beim Zuschauer selbst. Ein bedenklicher Niveauverlust ist erst dann zu beklagen, wenn andere, qualitative Werte verletzt werden.

\section{Vom ,Entertainment‘ zum ,Infotainment'?}

Mit „schlechter Unterhaltung" verhält es sich wie mit dem vielzitierten schlechten Geschmack: Jedermann fühlt sich als Experte, verurteilt pauschal das sinkende Niveau des Fernsehens - und bleibt, sobald eine Begründung verlangt wird, die Antwort schuldig. In Bereichen wie Information und Wissenschaft gibt es Kriterien, an denen sich richtig und falsch, schlecht und gut bemessen lassen. Wo aber liegen die Kriterien für gute Unterhaltung? Oder ist sie einfach Geschmacksache? Wenn dem so wäre, könnte man alles für gute Unterhaltung halten, was eben gefällt - und im Falle der Fernsehunterhaltung die Einschaltquoten allein zum Gütesiegel erheben. Das würde aber heißen, daß in Zukunft ein in Zeitlupe live im Fernsehen gesendeter Selbstmord - bei entsprechender Einschaltquote - zur besten Unterhaltungssendung des Jahres gekürt werden müßte.

Hier möchte ich auf die Thesen von Neil Postman zurückgreifen, um die Gefahren ausufernder Unterhaltung zu skizzieren. Gefahr droht durch FernsehUnterhaltung dann, wenn sie in alle Bereiche des Lebens eindringt und damit das Fernsehen dabei ist, „unsere Kultur in eine riesige Arena für das Showbusiness zu verwandeln" ${ }^{12}$ Fatal ist auch für Postman nicht die Tatsache, daß sich die Zuschauer unterhalten wollen bzw. das Medium Unterhaltung anbietet, sondern daß alles und jedes in der Form der Unterhaltung präsentiert wird. Wenn Fernsehen dabei zu einem rein kommerziellen Unterhaltungsmittel verkommt,

12 Neil Postman, a.a.O., 102. Siehe dazu auch meine Ausführungen in „Fernsehen am Wendepunkt", a.a.O., 70ff. 
das sich um die eventuell verletzten gesellschaftlichen Belange nicht kümmert, schließe ich mich den Warnungen vor einem übergreifenden Entertainment an. „Infotainment" oder „Reality-TV“ sind die Schlagworte, unter denen Information zu Unterhaltungszwecken mißbraucht wird.

Auch wenn es keine unanfechtbaren Normen für einen „Standard of Taste" gibt oder geben kann, gibt es doch Grenzen, die nicht überschritten werden dürfen. Auch bei noch so großem Publikumsinteresse muß die Privatsphäre des "Medienopfers" gewahrt bleiben, darf die Würde der Person durch das Recht auf freie Information nicht verletzt werden. Geschmacklos und gefährlich ist es zum Beispiel, wenn das Leiden von Menschen zur Unterhaltung mißbraucht wird, wie es z.B. durch Sendungen wie "Augenzeugen-Video" oder "Notruf" geschieht. Wenn durch sogenanntes "Reality TV" die Darstellung von "wirklicher" Gewalt zur Massenbelustigung benützt wird, sind die Grenzen des Zumutbaren überschritten. Auch das legitime Bedürfnis nach Unterhaltung rechtfertigt nicht, daß andere schützenswerte Rechte wie die Würde der Person einfach übergangen werden.

Wenn in Sendungen wie „Einspruch" das Aufeinanderprallen der Meinungen wichtiger, d.h. "unterhaltender" wird als als der Inhalt, widerspricht diese Form sogenannter Unterhaltung meiner Auffassung vom Fernsehen als Kulturgut.

Sensationsjournalismus, der die Realität als Krimi darzustellen versucht, liefert nur einen Nervenkitzel. ${ }^{13}$ Ideenreichtum ist auf lange Sicht auch in der Unterhaltung durch Reizerhöhung nicht ersetzbar. Auch diese Form der Ideenlosigkeit wird jedoch in Zukunft "Reiz-voll" im wörtlichen Sinne nur so lange bleiben, bis auch hier eine Gewöhnung und damit ein Desinteresse eingetreten ist.

Ein weiterer Gefahrenbereich liegt m.E. in der vermehrten Nutzung gewaltverherrlichender fiktionaler Filme wie "Rambo" oder "Terminator" im Unterhaltungsprogramm kommerzieller Sender. Die erhöhte Aggressivitäts-Bereitschaft durch "ge-reizte" Zuschauer ist dabei ebenso zu fürchten wie eine mögliche „Über-reizung“, die zur Abstumpfung und Passivität führen kann. Ein Übermaß von solchermaßen falschverstandener, Unterhaltung kann gefährlich werden, wenn sie durch Gewöhnung an Fernsehgewalt z.B. indirekt auch die Gewaltbereitschaft in der Gesellschaft erhöht. Hier muß eine Rückbesinnung auf die Verantwortung, die mit dem eigentlichen Unterhaltungsauftrag des Fernsehens verbunden ist, einsetzen, um einem drohenden Kulturverfall vorzubeugen. ${ }^{14}$ Nicht im Medium Fernsehen und seiner Unterhaltungsfunktion sehe ich die Gefahr des Kulturverfalls, sondern in seinem Mißbrauch.

13 Vgl. Winfried Goepfert, Infotainment. Unterhaltung als journalistisches Stilmittel, in: Bertelsmann-Briefe, Heft 128, Oktober 1992, 48-51.

14 Vgl. meinen Artikel „Zum Unterhaltungsauftrag des Fernsehens“, in: Dieter Prokop (Hg.), Massenkommunikationsforschung, Band 3: Produktanalysen, Frankfurt/M. $1977,481-492$. 


\section{Ausblick}

Abschließend möchte ich nochmals unterstreichen, daß sich Information, Unterhaltung und Kultur nicht wechselseitig ausschließen, sondern im Idealfall die einander ergänzenden Elemente ein- und derselben Sendung bilden. Unterhaltung - oft als das schwerste Fach des Fernsehens überhaupt bezeichnet kann sich nicht an allgemein verbindlichen Kriterien des Geschmacks orientieren, so daß für mich die tautologische Formel von Wolfgang Ernst nach wie vor überzeugt: „Unterhaltung ist das, was den Zuschauer unterhält." ${ }^{15}$ Der subjektive Maßstab ist letztlich die Akzeptanz des Programms durch den Zuschauer, die sich in der Einschaltquote niederschlägt. Hier ist die Emanzipation des Zuschauers am weitesten fortgeschritten, da letztlich nur er entscheidet, was ihn amüsiert. Spaß, Abwechslung und Entspannung sind unverfängliche Ziele. Im Übermaß genossen kann Unterhaltung aber auch zur Gefahr werden, insbesondere dann, wenn - wie Neil Postman mit Blick auf die U.S.-amerikanischen Verhältnisse kritisiert - alles und jedes, also auch Information und Kultur, zur bloßen Unterhaltung verkommt.

Ich teile jedoch die resignative Haltung vieler Film- und Fernsehschaffenden nicht, obwohl wir ihre Warnung ernst nehmen sollten. Dies kann unter anderem dadurch geschehen, daß wir uns auf die Urformen der Unterhaltung besinnen. Eine solche ist das Erzählen von Geschichten. In Geschichten wird das Erleben zum Beispiel. Im Erlebnis werden Anliegen vermittelt, in Märchen liegt immer auch ein Kern Wahrheit. Nicht abstrakte Theorie wirkt unterhaltend, sondern anschauliches Erzählen, das auch komplizierte Inhalte greifbar (also anschaulich) und begreifbar macht. Die Phantasie wird angeregt und letztlich auch die Bewältigung des Alltags erleichtert. So gesehen verhilft Unterhaltung nicht nur zur Entspannung und Erholung vom Alltagsstreß, sondern kann zur Orientierungshilfe in einèr sich ständig wandelnden Welt werden.

Ich vertraue darauf, daß im Überangebot der Unterhaltung in der derzeitigen Fernsehlandschaft ${ }^{16}$ die Chance liegt, daß auf seiten des Publikums bald ein Sättigungsgrad erreicht ist, der einer qualitätsbetonten Mischung von Witz, Humor, Spannung und bildenden Elementen auf lange Sicht größere Chancen

15 Im Original lautet die Formel: „Unterhaltung ist also, was unterhält.“, vgl. Wolfgang Ernst, Der Fernsehzuschauer und das Unterhaltungsangebot Fernsehen, in: Gerhard Prager (Hg.), Fernsehkritik, Unterhaltung und Unterhaltendes im Fernsehen, Mainz 1971, 54.

16 Beispielhaft ist auf die Ausstrahlung von etwa 35 Spielfilmen pro Tag für 1992 in Deutschland zu verweisen. Bei insgesamt 13.000 Sendeterminen für Filme - fast 5.000 mehr als 1991, - sinkt die Attraktivität dieses Genres für die Unterhaltung, da so viele Titel mit Niveau weder produziert noch eine derartige Quantität vom Zuschauer auch nur wahrgenommen werden können. 
einräumt als billigem „Klamauk“ oder dem Appell an ,dumpfe Triebe' des Menschen. Erotik-Shows verlieren ihren Reiz, wenn Tabus als solche nicht mehr wahrgenommen werden. Das Interesse an der ewigen Wiederkehr des Gleichen wird sinken.

Für das öffentlich-rechtliche Fernsehen wird es auch im Bereich der Unterhaltung in Zukunft darum gehen, Qualität mit Quote zu verbinden.

Auch bis zum Jahr 2000 und darüber hinaus wird sich das Bedürfnis der Zuschauer nach Unterhaltung nicht verringern, sondern eher noch verstärken. Für das öffentlich-rechtliche Fernsehen gilt dabei, daß auch in der Unterhaltung nicht ,die immer dickere Sau durch den Ort getrieben werden kann', um Aufmerksamkeit zu erzielen: vielmehr wird es darum gehen, Unterhaltungssendungen anregend, phantasievoll und einfühlsam zu gestalten, im Vertrauen darauf, daß der Geschmack der Zuschauer dafür sorgen wird, daß niveauvolle Unterhaltung letztendlich immer ihr Publikum finden wird.

SUMMARY: Television entertainment up to the year 2000: an area of conflict between high culture and everyday culture

Television entertainment may be defined as the opposite of boredom - and it is just as important for mankind as education and information. As it is the case with games, its value lies in itself. It corresponds to the audience's basic need for relaxation and rest after the stains of everyday life. The culture-critical disapproval of entertainment can be put down to an unsolved understanding of the cultural role of television: on the one hand there is a misjudgement of the nature of this mass medium aimed at large sections of the audience if one sticks to the elitist understanding of culture; on the other ratings and commerce should not become the sole quality standard of television entertainment. Against this semblance of alternative, the author sets the notion of "everyday culture" from which one can deduce the demand for a large variety on the programme. Thus, entertainment remains an integral part of every total programme (Grundversorgungsauftrag) and, being a part of the basic supply task, it may not be left in the hands of the commercial suppliers alone.

This contribution opposes the abuse of entertainment in the field of information and education which appears particularly at times when entertainment is extended to everything in television. Concerning the future of entertainment the author remains optimistic. As far as the audience's demands for higher standards of television entertainment are growing, programmes of quality will stand a good chance in the future, too.

RÉSUMÉ: Divertissement télévisé jusqu'à l'an 2000:

Divertissement tiraillé entre "culture" et "culture au quotidien"

Le divertissement télévisé se laisșe définir comme le contraire de l'ennui - et est aussi important pour l'homme que l'instruction et l'information. Comme pour le jeu, sa valeur se trouve en elle-même, elle correspond aux besoins de base des téléspectateurs à la recherche de détente, de délassement après le efforts de la vie quotidienne. Le refus 
critico-cuturel du divertissement se ramène à une compréhension inexpliquée de la notion de culture en ce qui concerne la télévision: D'un côté, on ne reconnaît pas que l'objectif de ce moyen audio-visuel est de s'adresser à une très large palette de téléspectateurs, de l'autre, on ne doit pas seulement considerer le taux d'écoute ou le commerce comme mesure de qualité du divertissement télévisé. A cette alternative spécieuse, l'auteur oppose le terme de "culture au quotidien" à partir duquel se laisse déduire l'exigence de diversité au niveau du contenu du programme. Le divertissement reste partie intégrante de chaque programme complet et ne peut, dans sa mission en tant que partie d'un apport essentiel („Grundversorgungsauftrag"), être laissé aux seuls agents commerciaux. L'article s'attaque à l'emploi abusif du divertissement dans le domaine de l'information et de l'instruction. Celui-ci apparaît tout particulièrement quand on étend le divertissement à n'importè quelle émission. En ce qui concerne l'avenir du divertissement, l'auteur reste optimiste. Comme on demande toujours plus au divertissement télévisé, le émissions de hauts niveaux ont de bonnes chances dans l'avenir.

RESUMEN: Los programas de entretención en la TV hasta el año 2000: Entre la cultura alta y la cultura cotidiana

Los programas de entretención en la TV se pueden definir como lo contrario del aburrimiento y son también tan importantes para la gente como la formación y la información. Su valor está, como en los juegos, en si mismo; corresponde a la necesidad de los televidentes de distención y descanso de los esfuerzos del día. El rechazo críticocultural de estos programas se puede retrotraer a una concepción confusa de cultura frente a la TV: por un lado no se comprende la amplitud de las capas sociales a las que está dirigido este medio de masas; por otro lado, si se perservera en una concepción elistista de la cultura, no pueden ser solo la cuota de telespectadores o el comercio la medida de la calidad de los programas de entretención. En contra de esta alternativa ilusoria, el autor propone el concepto de „cultura cotidiana“, del que se deduce la exigencia de un contenido variado y diverso en la programación. Los programas de entretención permanecen así siendo parte componente de toda programación y como parte fundamental de ésta no puede ser dejados solo a la oferta comercial ("Grundversorgungsauftrag“). El artículo toma posición contra el mal uso de la entretención en el campo de la información y en el educativo, que especialmente aparece cuando la entretención se extiende a todo. En lo que toca al futuro de los programas de entretención, el autor se muestra optimista. Las exigencias y espectativas a los programas de entretencion estan cresciendo y los programas de alto nivel tienen buena chance. 